\title{
Supporting the running and analysis of trials of web-based behavioural interventions: the LifeGuide
}

\author{
Yang Yang ${ }^{1}$, Adrian Osmond ${ }^{1}$, Xiaoyu Chen ${ }^{1}$, Mark Weal $^{1}$, Gary Wills ${ }^{1}$, David De Roure ${ }^{1}$ \\ Judith Joseph ${ }^{2}$ and Lucy Yardley ${ }^{2}$ \\ ${ }^{1}$ School of Electronics \& Computer Science, \\ University of Southampton \\ \{yy4, aco, mjw, gbw, dder\}@ecs.soton.ac.uk \\ ${ }^{2}$ School of Psychology, \\ University of Southampton \\ \{J.Joseph, L.Yardley\}@soton.ac.uk
}

\begin{abstract}
Behavioural interventions - packages of advice and support for behaviour change - are one of the most important methodologies and technologies employed by social scientists for understanding and changing behaviour. A typical web-based behavioural intervention study includes the designing, deploying, piloting and trialling of the intervention as well as data analysis. We have developed a research environment named LifeGuide, which covers the full scope of this process, enabling social scientists to carry out intervention studies with minimal technical expertise. In this paper, we present how the LifeGuide can assist and accelerate intervention research, particularly focusing on supporting the running and analysis of trials of webbased behavioural interventions along with the case study of an intervention that has been developed within the LifeGuide.
\end{abstract}

Keywords-Behavioural intervention;

\section{INTRODUCTION}

There are many times where change in one's behaviour could be beneficial to both an individual and society. For instance, persuading people to cease smoking, to wash hands to prevent pandemic flu, to avoid unprotected sexual intercourse, or to eat a healthy diet could potentially improve the health of a population [1]. Behavioural interventions are packages of tailored advice and support for behaviour change, which are known as one of the most important methodologies and technologies adopted by social scientists for understanding and changing behaviour [2]. Traditionally, behavioural interventions have been delivered face-to-face with experts in the related field. The major problem of this approach is that it is very costly and resource intensive. Moreover it is impossible to provide everyone with 24 hour access to personal guidance on managing all their problems.
The World Wide Web, for the first time, provides a platform to reach millions of people without the restrictions of location and time. In view of this huge potential, there has been a trend for more and more behavioural interventions to be delivered through the Internet. Web-based behavioural interventions (WBBIs) typically transform the face-to-face process into interactive and adaptive web applications - where experts set a series of questions that appear in interactive web pages. Tailored advice is then displayed based on the intervention user's responses.

Although the web offers a promising way of delivering interventions, WBBI development is relatively slow. Since WBBIs are not as straightforward as standard websites, social scientists often require assistance from software engineers to design and deploy their interventions. This introduces the extra cost of hiring personnel and once an intervention is deployed, it cannot be easily modified. Many commercial applications that could potentially be used for creating interventions have been shown to be inflexible for social science research [3]. Therefore, researchers need to develop WBBI applications and analyse the data of each intervention individually. This vast duplication of effort in producing similar WBBIs has limited the number of interventions that can be developed and evaluated, thereby limiting the accumulation of knowledge surrounding behavioural intervention research. All of these barriers hinder intervention researchers in developing and testing WBBIs.

A WBBI study includes the designing, deploying, piloting and trialling of the intervention as well as data analysis. We have developed a research environment named LifeGuide, which covers the full scope of this process, enabling researchers to carry out an intervention study with minimal technical expertise. Interventions created within LifeGuide can be shared or reused within the research community to devise evolving interventions. Systematic collection of trial 
data across multiple interventions will provide an important new resource for researchers, enabling them to integrate data and carry out large-scale data analysis to answer further research questions.

The LifeGuide was co-designed by both computer scientists and social scientists. In order to ascertain the system requirements, we have developed a WBBI named Internet Doctor along with developing the LifeGuide. This intervention provides users suffering from colds and influenza with tailored advice to enable them to manage their symptoms. To date, qualitative and quantitative studies of this intervention have been completed and some of the results have been published $[4,5]$. In this paper, we present how the LifeGuide research environment can be beneficial to intervention studies, particularly focusing on trialling an intervention and analysing its data along with the case study of the Internet Doctor.

\section{BACKGROUND LITERATURE}

Behavioural interventions are traditionally delivered face-to-face by domain experts, such as a therapist in a given field. An intervention will typically take place over a period of time, during which tailored advice will be given to a client. This has been the overwhelmingly dominant method of intervention delivery. It has many benefits as it allows an expert to get to know the people they are helping and make the advice that they give completely personalised to each individual. Printed materials (e.g. booklets and manuals) are often used in this approach to disseminate motivational behavioural interventions. The efficacy of the intervention is measured by evaluating whether or not clients change their behaviour in response to the advice.

WBBIs transform the face-to-face approach into interactive and adaptive web applications. In a typical form of WBBI, experts set a series of questions that are presented as interactive web pages and tailored advice is displayed for the subject based on how they respond to questions. It is highly unlikely that WBBIs will ever replace the role of the domain expert to provide tailored advice for intervention users. Nevertheless, in many situations WBBIs can usefully complement and supplement expert advice, and offer their own benefits compared with the traditional method. Based on Fogg's persuasive technology theory [6] and several systematic studies of WBBIs [7, 8], we summarise many of their advantages below:

- WBBIs can provide accurate and persistent advice based on given information and logic of inference, where social experts can get tired, discouraged or frustrated.
- WBBIs can access large volumes of information stored in databases. These can be expanded and updated instantly which is far beyond the capabilities of human beings.

- WBBIs can be extended by various technologies, such as streaming media, internet telephony, video games and many other forms of computer applications. They can therefore provide adaptive interaction in various modalities to produce optimum persuasive impact.

- WBBIs enable anonymity and reduce stigma. Some clients find that some behavioural interventions (e.g. substance abuse and sexual behaviour interventions) can be too sensitive to be carried out in a face-to-face form.

- WBBIs are ubiquitous and cost-effective. They can be accessed by millions of users beyond the restrictions of time and location. This opens up the opportunity to provide an individual with 24 hour support for managing all aspects of their lives.

Since the many benefits of WBBIs have been realised, there has been a trend for more and more behavioural intervention studies to be carried out online. However, at this stage in time, due to the lack of a generic intervention authoring tool, researchers need to program the application infrastructure and data analysis for each WBBIs individually. The potential of WBBIs is therefore severely restricted. Some commercial software packages 12 developed lately enable researchers to customise the content for a particular intervention they wish to create. These support the production of websites, but rarely include any ability to run a randomized, stratified trial or to collect and analyse results in a rigorous manner. In this approach, data collection may only be possible by reading through web server logs, which is problematic and often involves bespoke solutions. The LifeGuide infrastructure is designed within this context to conveniently help social scientists to carry out intervention studies.

In order to develop a behavioural intervention research environment for social scientists, we have investigated many other e-science infrastructures and environments. Particularly, we studied the design approach of the ${ }^{m y}$ Experiment Virtual Research Environment (VRE) - a system for collaboration and sharing of experiments, where De Roure and Goble [9] proposed six principles of design for e-science applications to empower scientists. The LifeGuide system was co-designed by both computer scientists and social scientist and the development process was completely user-centric and user-driven.

\footnotetext{
${ }^{1}$ www.healthmedia.com

2 www.changetech.no
} 


\section{THE DEVELOPMENT OF LIFEGUIDE}

A web-based intervention study often takes several phases. Figure 1 illustrates the web-based intervention research cycle. Firstly, social scientists need to explore the relevant theory to ensure the best choice of intervention and identify the components of the intervention. Once an intervention and its study are designed, graphical designers create the look and feel of web pages and computer programmers then transform the web pages into an adaptive web application. It can then go on to a pilot study, which often involves testing the feasibility of delivering the intervention and acceptability to providers and intervention users. This often results in a lot of changes in the intervention. When it reaches a relatively mature stage, it is published as a trial. Participants can then access the intervention online. All their data is recorded in the web server data logs for further data analysis. Once data in the final study has been analysed, modifications to the hypothesis can be made, forming another study and the cycle begins again.

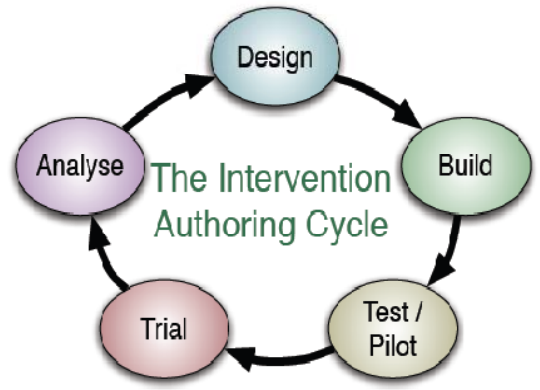

Figure 1. The intervention research cycle.

We aimed to develop a research environment to cover the full scope of this process, enabling a researcher to carry out an intervention study without the mediation of software engineers. Based on this, three major requirements were identified:

i) The system should enable social scientists with no graphic design experience to create web pages;

ii) The system should allow social scientists with no programming experience to program adaptive interventions and studies;

iii) The system should provide a research environment within which social scientists feel familiar carrying out their studies.

We adopted a co-design process where computer scientists and social scientists worked together at each stage of the design and development. This has ensured valuable early feedback and regular reviews and refinements to be made. At an early stage of the development, we used extensive expert consultation through workshops and the Internet to obtain researchers' views of how to make the software fit all of their requirements. We investigated a series of web design applications and put a mock-up system together. Social scientists were particularly keen that LifeGuide should be able to create web pages just as easily as presentation slides can be created in Microsoft ${ }^{\circledR}$ PowerPoint $^{\mathrm{TM}}$. This has been used as a reference by our developers to measure the usability of our prototype system.

There are two set of users of LifeGuide: researchers and intervention users. The researchers use LifeGuide to authoring the interventions. The intervention users will, at a later time, access those interventions created by the researcher via the Web. Therefore, LifeGuide system was designed to have two major components: a Desktop Authoring Tool for creating and editing intervention applications and an Intervention Manager Web Portal for deploying the intervention and record and analyse the data of the intervention participants. Researchers firstly create an intervention on their local computer in the authoring tool, then export and upload it to the intervention manager web portal. A trial study can be then carried out online. Once an experiment is completed, the data can be exported from the portal for further analysis.

From a functional perspective, LifeGuide can be described as providing two major services:

i) A platform for performing WBBIs - this includes the process of designing, building and deploying the intervention

ii) A research environment that assists social scientists in carrying out intervention studies, which may include the piloting, trialling and data analysis of the intervention.

To achieve both of these, we have developed an intervention along with developing the LifeGuide. This intervention functioned as a probe to ascertain the requirements to design the research environment. The intervention named the Internet Doctor was designed to advise users whether there were medical indications that they should consult their doctor for their current symptoms of influenza and the common cold, and to provide information that could help them self-care if appropriate. In the following section, we will briefly introduce how an intervention is designed and deployed within LifeGuide along with the case study of the Internet Doctor and in the later section we will mainly focus on discussing how an intervention study is carried out in the research environment which we specially tailored for social scientists

\section{A. A platform for performing web-based behavioural interventions}


The Desktop Authoring Tool enables researchers to create and edit interventions with minimal technical expertise. The application offers a "What You See is What You Get" web page editor, where the user can drag and drop a set of customisable intervention components onto a webpage. For example, to create choice based questions, the user can choose the "multiple choice interaction" component by simply clicking, and then customise the question and choice content. We also provide a set of multimedia components such as video and flash animation, which can be used by the authors to demonstrate correct behaviour to their target intervention users or to design more graphically appealing interactions. Figure 2 illustrates the user interface of the Desktop Authoring Tool. At the bottom of the page we have provided a list of intervention components, such as text boxes, images, videos, buttons, tables and so on. On the right hand pane, an adaptive menu is displayed, which contains different menu options based on the selected item on the page. In order to assist researchers in developing more professional looking webpages, we also offer a set of webpage themes and templates for researchers to customise and design their own intervention web applications.

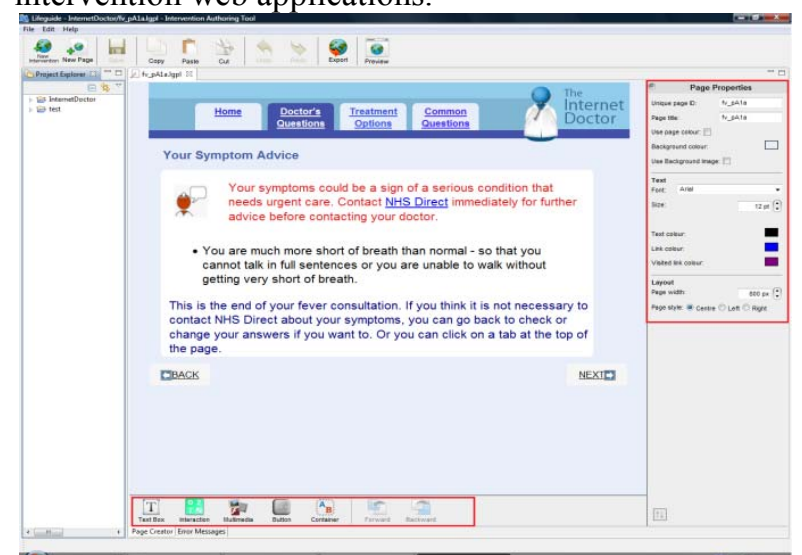

Figure 2. The Desktop Authoring Tool editing an Internet Doctor page.

Once webpages are created with the tool, they are then linked together by an intervention logic. This is a set of instructions to determine the order in which webpages should be displayed, as well as how the questions and tailored advice are presented and evaluated. This intervention logic can be represented in many different ways, such as graphically or via a scripting language. The choice of the method to represent the logic of an intervention will depend on the personal preference of the researcher in question. In LifeGuide, both graphical representation and logic scripting language approaches have been investigated to enable authors to express the intervention logic, similar to the workflow systems, such as Meandre ${ }^{3}$, where a scripting language called zigzag with the logic expressed in $\mathrm{RDF}^{4}$ and visualised as workflow graphs. The first approach to represent the intervention logic in Desktop Authoring Tool used logic scripting language.

It was a challenge to design a scripting language which could enable social scientists with no programming experience to program adaptive interventions. From the social scientists' point of view, they would like to express logic in a concise English form. Ideally, they could "script" a paragraph of the logic of their intervention and the LifeGuide would do the rest of the job. However, from the computer scientist's perspective, we need to avoid semantic ambiguity when designing the syntax. Therefore, the line we drew in between these two requirements was to make sure our language was understandable by both the human and the computer. The logic scripting language's syntax is based on plain English and the order of expressions is designed to be similar to the order of concise English grammar. For instance, "show advicePage if (page1.question = 'yes')" means display the page named "advicePage" if the user responds "yes" to the question on page 1. Figure 3 demonstrates the editor we created for social scientists to program their interventions, where the code auto-completion feature is developed to assist users in scripting.

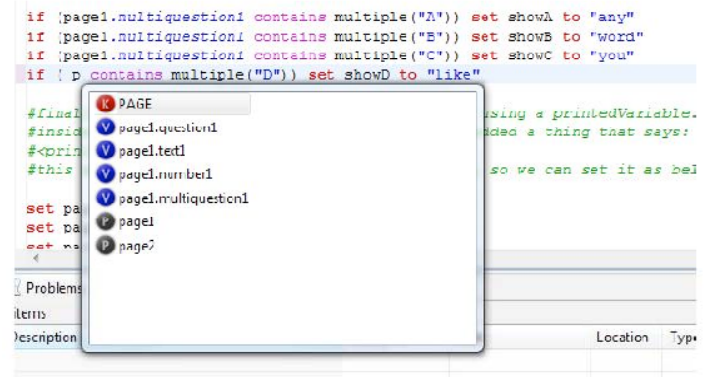

Figure 3. The logic editor's auto-completion of a demo intervention.

The scripting language we designed in LifeGuide mainly serves two purposes: one is to program the intervention itself, the other is to enable researchers to program aspects relating to how their trial will be carried out. In order to measure the effectiveness of an intervention trial, Randomized Controlled Trials are commonly used to randomly allocate different interventions to participants, where participants are often stratified into groups to be studied and compared. We have created a set of commands for these needs. First of all, in order to manage the participants of the intervention, we need to provide a series of user

\footnotetext{
${ }^{3}$ https://kepler-project.org/

${ }^{4}$ http://www.w3.org/RDF/
} 
account management features. Commands such as "make a new user" and "check if user already exists", were designed to enable the researcher to manage user accounts with a few lines of code. Once participants' accounts are created, they can be stratified into groups based on criteria set by researchers. The code snippet below illustrates the stratification of participants based on the age and gender. If participants are males and under 18 , they will be randomly allocated into two different versions of the intervention. The randomisation picks a random number that is either 0 or 1 with $50 \%$ chance of either outcome. If the number is 0, participants will be directed to "intervention1". Similar code could be used to stratify the participants into other groups based on different genders and ages.

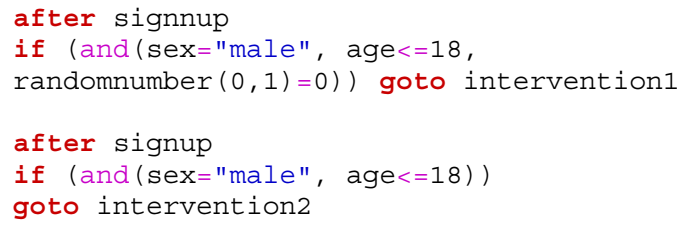

In order to support sustained behaviour, follow-up studies of an intervention are often required. The access of an intervention can be controlled over time. For instance, one can design an intervention that can allow participants to view different content on a week by week basis. We created a "send email" command, which can automatically send out emails to a user at a given time. This could also be used to remind the user to take part in the follow-up studies. There is also a command designed to check if a user has viewed a particular webpage. When the user receives the reminder email and accesses the site again, it will allow them to see only pages that they haven't seen before. We also plan to provide text messaging in LifeGuide, which could be used in conjunction with or as an alternative to email.

Once a WBBI is designed, it often goes on a pilot study to test the feasibility of delivering the intervention and acceptability to providers and intervention users. In the Internet Doctor case, the author particularly wanted to carry out a study which would randomise participants into two groups: one group would be directed to a full "more interactive" version and another one would be directed to a "less interactive" version. This study aimed to examine quantitative indices of the usability of two different formats for presenting the intervention. There were two follow-up emails sent out in this study. One email was sent out 24 hours after registration, and the other 4 weeks after registration. The following code snippet illustrates simple randomization, where if the output of a random number function is 0 , it allocates users into the full version group and directs the user to the more interactive site. Otherwise, the user will be allocated to the less interactive site. The follow up emails will be sent out after 24 hours (86400 seconds) and 4 weeks with different content.

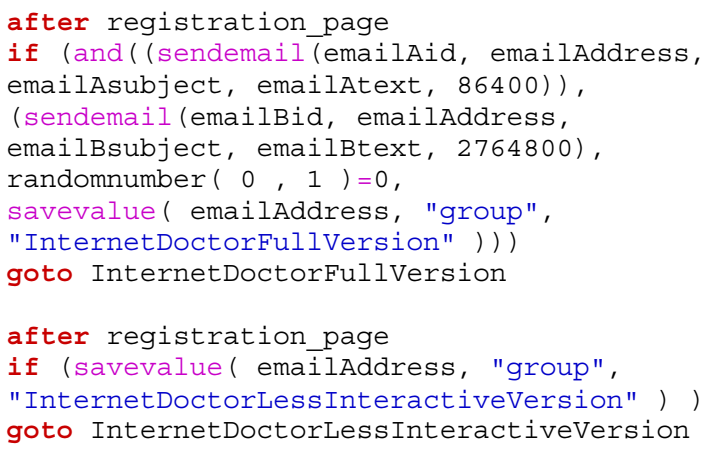

When authors create interventions inside the Desktop Authoring Tool, they can be exported as an intervention package to be uploaded to the Intervention Manager Web Portal. Participants can then access the intervention through a web browser from anywhere in the World.

\section{B. A behavioural intervention research environment for social scientists}

WBBIs are often run as trials once they reach a mature stage. The Intervention Manager Web Portal is particularly designed for deploying, trialling and analyzing intervention data. Figure 4 illustrates the user interface of the Intervention Manager Web Portal. When researchers upload their intervention packages to the portal, the Intervention Manager offers the option of setting the start and expiry dates for a trial as well as providing a name that will form part of the public URL to the intervention. After deploying the intervention, the researcher can send a recruitment email to potential participants along with the URL. All participants' intervention usage data is collected by the Intervention manager. As researchers may wish to view the collected data from a number of perspectives in order to understand its nature, we provide a set of data analysis tools to assist intervention studies.

The "information section" (see Figure 4 item 1) displays general information about an intervention such as the total number of visits of anonymous and logged in users. Item 2 lists each individual 


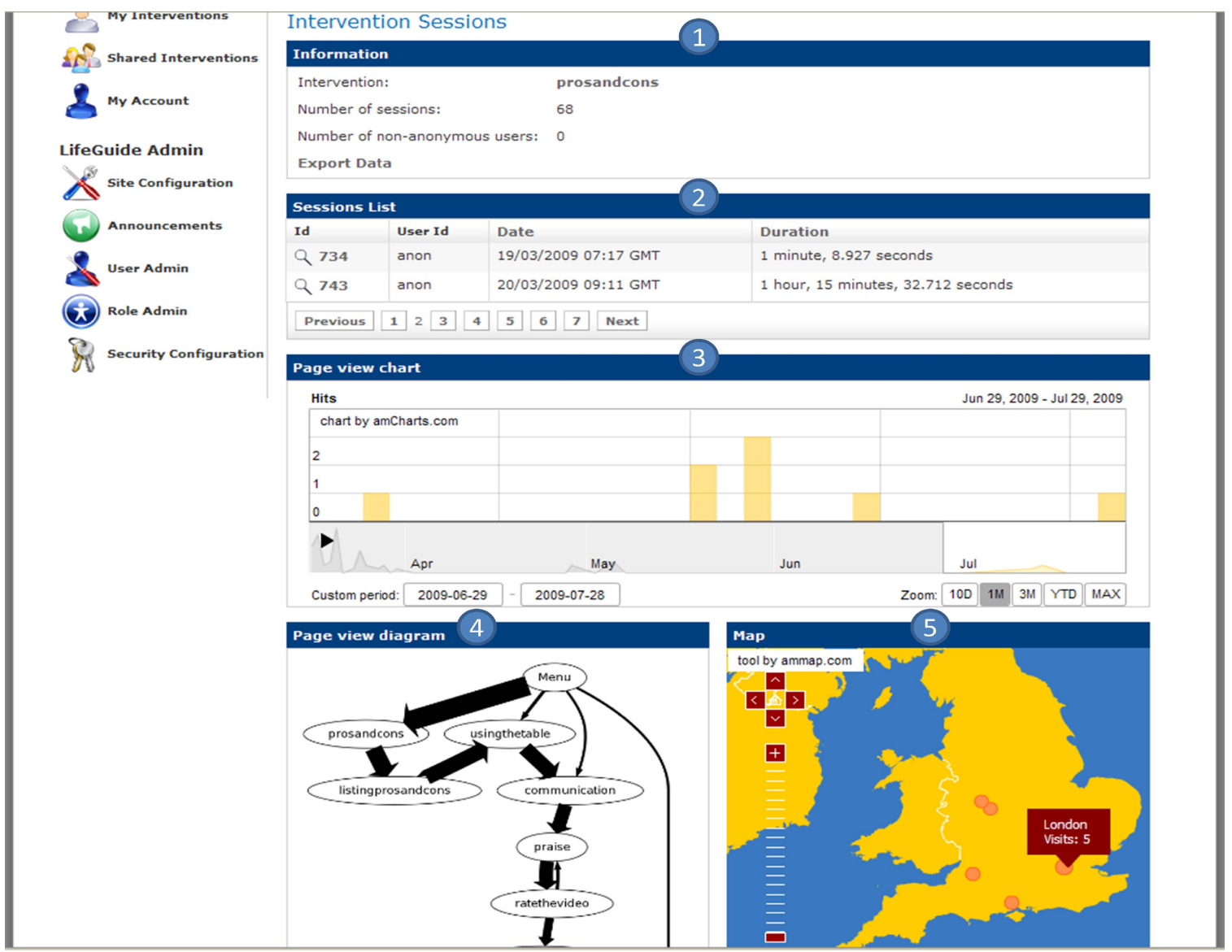

Figure 4. Intervention Manager Web Portal

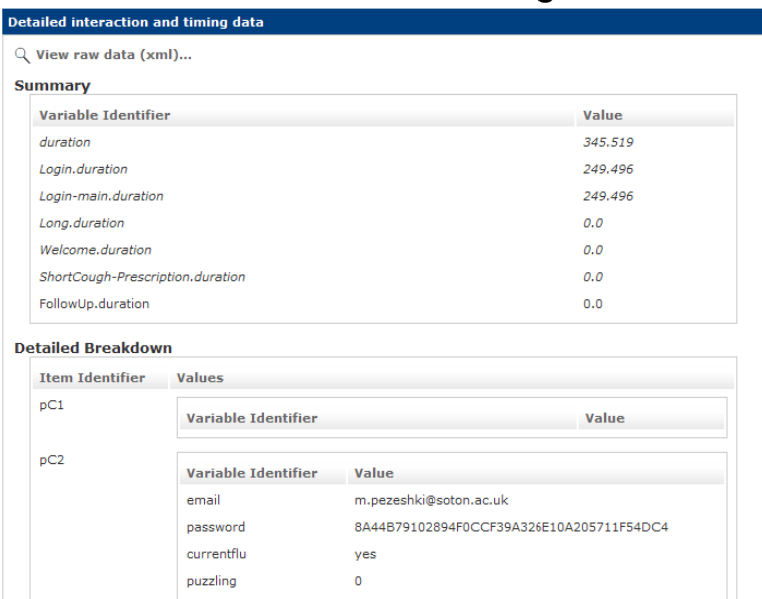

Figure 5. Intervention data table

participant's user id, access time and the duration spent on the intervention. All intervention participants' data from their responses on every single question to the order of pages viewed and the amount of time spent on each part of the intervention are recorded by the Intervention Manager. Each participant's data can be visualised in a table as shown in Figure 5. In the "summary" section, the aggregated data information enables researchers to have a quick overview of the data and identify possible patterns. In the "detailed breakdown" section, detailed data such as a user's response to a particular question are categorized based on each individual intervention web page. The data can be used to identify the effectiveness of an intervention and its impact on an individual basis.

Figure 4 item 3 shows the intervention Page View Chart, which statistically displays the number of visits of an intervention over a period of time. The scale of the graph can be customised between 10 days and the year-to-date. Regular peaks in this graph could indicate that participants are continuing to take part in the intervention. It can also be utilized to identify the usefulness or the popularity of an intervention at a given time as well as trends in usage. For instance, the access of a pandemic flu related intervention may dramatically increase while the flu is spreading over a population. Figure 4 item 5 - the intervention access map - graphically displays where intervention users were geographically located. To evaluate a sample size over a population, many intervention studies are restricted to a particular location and the intervention users may be geographically stratified. The intervention access map offers a clear overview of the distribution of intervention users. 
The participant's navigation path can be important to many intervention studies. Figure 4 item 4 displays the aggregated navigation path of the intervention, where the thickness of an arrow represents the access frequency level. The most popular navigation path can be easily identified through the graph. By linking this understanding to outcomes of the intervention, the researcher can determine what intervention components are most effective. In the qualitative study of an intervention, the web navigation path can be analysed in usability testing to identify any potential problems with the intervention design. Within LifeGuide, researchers can also go into more detail to analyse an individual's navigation path. Figure 6 illustrates the navigation path of a participant. The lengths of time that the participants spent interacting with web pages are labelled on the graph.

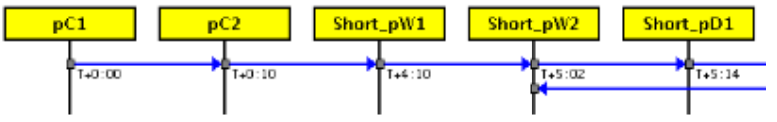

Figure 6. The navigation path of an intervention participant

All data recorded by the Intervention Manager can be exported from the web portal in the Excel file format for further data analysis. The architecture of the server application is shown in Figure 7. The data recorded by the Intervention Manager consists of two main parts: session information (e.g. users' IP addresses) and intervention data (e.g. the responses of users to questions). During the data analysis of the Internet Doctor, researchers found that the exported data from LifeGuide can be overly lengthy. This was improved by allowing the users to select which variables they wanted to appear in their export.

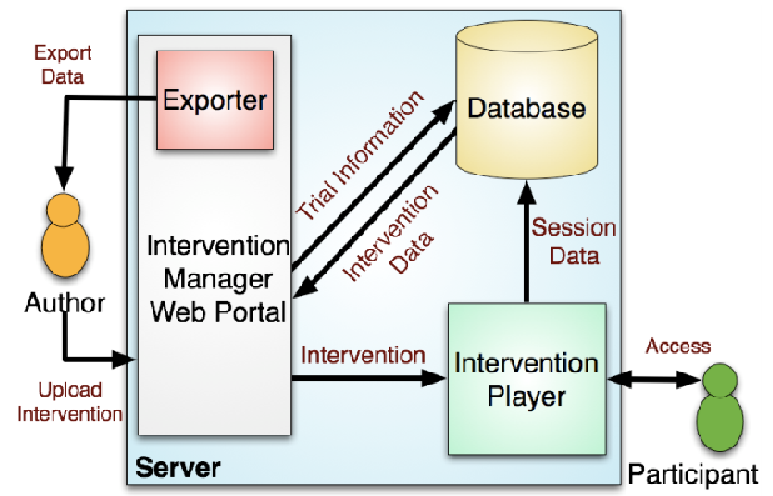

Figure 7. Architecture of the server application

On top of all of this, the Intervention Manager Web Portal also provides several social features such as a group mailing list, a wiki and message boards to enable communication between both intervention authors and users.

\section{TOWARDS OPEN BEHAVIOURAL INTERVENTION RESEARCH}

WBBIs are often designed based on a number of theories. For each intervention component, there are theories and studies to support its design decisions. From this perspective, WBBIs are knowledge assets. However, the benefits of WBBIs have not been well utilized in the past - we have seen many duplicated or overlapping intervention studies. For instance, smoking cessation interventions have been carried out many times ${ }^{5}[10]$, requiring design, building and piloting of the intervention every single time. Using LifeGuide - a generic intervention authoring system, opportunities are opened up for recycling old interventions and reinventing them for different purposes. All interventions generated by LifeGuide will form an intervention repository and researchers can develop evolving interventions based on previously authored interventions. Since ${ }^{m y}$ Experiment VRE, an e-science social network application, offers a promising way of sharing experiment workflows between scientists and it provides a large set of communication tools, it is our plan to integrate LifeGuide with ${ }^{m y}$ Experiment to enable social scientists to share their own intervention in a similar fashion to sharing workflows.

There are two social networks behind the behavioural intervention community: i) an intervention authors network; ii) an intervention users network. Both networks can be utilized for different purposes. Intervention research often involves qualitative studies, which are used for the initial elicitation of stakeholder needs and desires, assessment of intervention users' and experts' views regarding the content and format of interventions [11]. To digitalise this process is to enable the members of the intervention network to comment on each other's intervention components. The intervention user network will naturally form peerto-peer support groups and it can also be utilized for researchers to recruit the participants for intervention studies.

Based on the REST Web Service API of ${ }^{m y}$ Experiment, an intervention can be easily retrieved and shared. Since LifeGuide is an open source research platform, its server application can be downloaded and customised for individual use. For e-health related interventions, researchers can apply their own data protection policy. The decentralised data of refined intervention experiments can be integrated into a larger data set. The linked data movement, emerging from the Semantic Web community, can be applied maximize

\footnotetext{
${ }^{5}$ www.ceasetobacco.org
} 
the sharing and reuse of data. As web-based behavioural interventions become widely used in many aspects of life, the integrated data will eventually form a foundation for "population laboratories" in the future. The semantically linked data can then be utilized to carry out more powerful analysis to answer new research questions which have hitherto not been possible.

\section{CONCLUSIONS}

The LifeGuide research environment provides a platform to author and host web-based behavioural interventions and to assist researchers in carrying out their intervention studies. The system covers the full process of the intervention research cycle - from the designing, building and piloting to trialling and analysing of data. To date, there are a number of interventions under development using LifeGuide. We have conducted workshops in a number of institutions, demonstrating our system and a total of 144 researchers have joined the LifeGuide network. Although the LifeGuide has demonstrated immediate benefits to the research community, some challenges remain. Despite the fact that the majority of LifeGuide users can create interventions with simple logic, we have observed a difficulty in creating interventions with complex logic. The LifeGuide learning materials are currently under-development and a graphical programming method is also under investigation. The set of data analysis tools we have provided in LifeGuide could be improved by providing more elaborate information (e.g. to display the percentage of the distribution of the participants on the intervention map) and more interactive ways of browsing data. Since all intervention data can be exported in a standard format, some common data analysis procedures could also be further automated.

Interventions created within LifeGuide and their results can be shared or reused within the research community to devise evolving interventions. The data for each intervention could be potentially integrated to carry out large-scale data analysis to answer further research questions. Looking ahead, it is in our plan to integrate LifeGuide with the existing e-science virtual research environment to embrace more open behavioural intervention research.

\section{ACKNOWLEDGEMENTS}

The LifeGuide project is supported by the Economic and Social Research Council (grant number: RES-14925-1069). The development of Virtual Research Integration Collaboration project is funded by JISC.

\section{REFERENCES}

[1] S. Michie and C. Abraham, "Interventions to change health behaviours: evidence-based or evidenceinspired?," Psychology \& Health, 2004.

[2] L. Yardley, A. Osmond, J. Hare, G. Wills, M. Weal, D. d. Roure, and S. Michie, "Introduction to the LifeGuide: software facilitating the development of interactive internet interventions," in Symposium: Persuasive Technology and Digital Behaviour Intervention Edinburgh, Scotland, 2009.

[3] A. Osmond, J. Hare, J. Price, A. Smith, M. Weal, G. Wills, Y. Yang , L. Yardley, and D. De Roure, "Designing authoring tools for the creation of on-line behavioural interventions.," in Proceedings 5th International Conference on e-Social Science, Cologne, Germany, 2009.

[4] J. A. Joseph, L. Yardley, J. Hare, A. Osmond, Y. Yang, M. Weal, and G. Wills, "Application of the LifeGuide: The development and quantitative analysis of the 'Internet Doctor'. ," in Proceedings 5th International Conference on e-Social Science, Cologne, Germany., 2009.

[5] L. G. Morrison, J. A. Joseph, P. Andreou, and L. Yardley, "Application of the LifeGuide: A think-aloud study of users' experiences of the 'Internet Doctor'." in Proceedings 5th International Conference on e-Social Science, Cologne, Germany., 2009.

[6] B. J. Fogg, J. Grudin, J. Nielsen, and S. Card, Persuasive Technology: Using Computers to Change What We Think and Do: Science \& Technology Books, 2002.

[7] F. Griffiths, A. Lindenmeyer, J. Powell, P. Lowe, and M. Thorogood, "A systematic review of the published literature. Why are health care interventions delivered over the internet? ," Journal of Medical Internet Research, 2006.

[8] W. Dean, P. Carmen, H. William, S. Rob, and M. Eva, "The effectiveness of Web-based vs. non-Web-based interventions: a meta-analysis of behavioral change outcomes. ," Med Internet Res., 2004.

[9] D. De Roure and C. Goble, "Software Design for Empowering Scientists.," IEEE Software, vol. 26, pp. 8895, 2009.

[10] T. N. Chirikos and T. A. Herzog, "Cost-effectiveness analysis of a complementary health intervention: the case of smoking relapse prevention," Int. J. Technol. Assess. Health Care, vol. 20, pp. 475-480, 2004.

[11] C. Pagliari, "Design and evaluation in eHealth: challenges and implications for an interdisciplinary field.," Journal of Medical Internet Research, vol. 9, 2007. 